\title{
FMRI Responses to Hyperoxia in Congenital Central Hypoventilation Syndrome
}

\author{
MARY A. WOO, PAUL M. MACEY, KATHERINE E. MACEY, THOMAS G. KEENS, \\ MARLYN S. WOO, REBECCA K. HARPER, AND RONALD M. HARPER \\ School of Nursing [M.A.W.], Department of Neurobiology [P.M.M., K.E.M., R.K.H., R.M.H.], University \\ of California, Los Angeles, Los Angeles, California 90095, Childrens Hospital Los Angeles [T.G.K., \\ M.S.W.], Los Angeles, California 90027
}

\begin{abstract}
Congenital Central Hypoventilation Syndrome (CCHS) patients show partial retention of peripheral chemoreception despite impaired ventilatory responses to $\mathrm{CO}_{2}$ and hypoxia. The condition allows examination of central responses to hyperoxia, which minimizes afferent traffic from peripheral chemoreceptors. We used functional magnetic resonance imaging to assess blood oxygen level-dependent signals over the brain during a baseline and subsequent 2-min hyperoxia $\left(100 \% \mathrm{O}_{2}\right)$ period in $14 \mathrm{CCHS}$ and 15 control subjects. After partitioning gray matter and correcting for global effects, the images were analyzed using volume-of-interest time trends followed by repeated-measures ANOVA and conventional cluster analyses. Respiratory rates initially (first 20 s) fell in CCHS, but rose in control subjects; CCHS heart rate increased in the first minute, and then decreased in the second minute, as in controls, but with muted rise and extent of decline. Multiple sites within the cerebellum, midbrain, and pons responded similarly to the challenge in both groups. Response patterns differed early in the right amygdala, paralleling initial respiratory pattern deficits, and late in the right insula, concomitant with cardiac rate differences. Signals also differed between groups in the medial and anterior cingulate, hippocampus, head of caudate, and lentiform nuclei, as well as pontine and
\end{abstract}

\section{ABSTRACT}

midbrain structures and regions within the superior temporal and inferior frontal cortical gyri. The findings emphasize that structures that can alter respiratory timing, such as the amygdala, and modulate sympathetic outflow, such as the right insula, are deficient in CCHS. Medullary and pontine areas targeted by PHOX2B expression are also affected. (Pediatr Res 57: 510518, 2005)

$\quad$ Abbreviations
BOLD, blood oxygen level dependent
CCHS, Congenital Central Hypoventilation Syndrome
EPI, echo planar imaging
fMRI, functional magnetic resonance imaging
FOV, field of view
MNI, Montreal Neurological Institute
MRI, magnetic resonance imaging
NTS, nucleus of the solitary tract
RMANOVA, repeated measures ANOVA
SE, standard error
TE, time to echo
TR, repetition time
VOI, volumes of interest

In addition to impaired ventilatory responses to $\mathrm{CO}_{2}$ and deficient breathing drive during sleep (1), patients with CCHS show inadequate breathing and cardiovascular responses to elevated or lowered levels of $\mathrm{O}_{2}(2-5)$. The mechanisms involved in altered sensitivity to $\mathrm{O}_{2}$ in CCHS remain unclear, because some degree of both central and peripheral chemoreception apparently is retained in the syndrome. Respiratory rates increase to hypoxia and decrease to hyperoxia $(3,5,6)$, although both the respiratory and cardiovascular response patterns to challenges are distorted from the normal condition.

Received August 30, 2004; accepted November 15, 2004.

Correspondence: R.M. Harper, Ph.D., Department of Neurobiology, University of California at Los Angeles, Los Angeles, CA 90095-1763; e-mail: rharper@ucla.edu

Supported by the National Institutes of Child Health and Human Development Grant HD-22695

DOI: 10.1203/01.PDR.0000155763.93819.46
Determining the neural mechanisms underlying response deficits to differing $\mathrm{O}_{2}$ levels in CCHS is a significant issue for reasons other than the obvious respiratory implications: CNS responses to hypoxia or hyperoxia recruit the sympathetic nervous system to, among other cardiovascular actions, preferentially alter perfusion of the periphery. CCHS patients show substantial deficits in both sympathetic and parasympathetic components of autonomic nervous system control, which are manifested by such signs as extreme reduction in respiratoryrelated heart rate variability during sleep (7), diminished blood pressure "dipping" during the night (8), syncope to relatively mild provocation (9), a high incidence of cardiac arrhythmia (10), intolerance to heat and cold, and poor control of sweating (11). The autonomic deficits have the potential to modify compensatory cardiovascular changes and organ perfusion to alterations in $\mathrm{O}_{2}$ levels. Respiratory and cardiac rate and 
variability responses to hyperoxia in CCHS suggest deficiencies in both early and late physiologic responses, with particularly profound effects on rapid-variation aspects, as reflected in breath-by-breath heart rate changes (respiratory sinus arrhythmia).

A principal consequence of administering $100 \% \mathrm{O}_{2}$ is an effective suppression of peripheral chemoreceptor input (12). If peripheral chemoreception is at least partially intact in CCHS, the suppression of afferent chemoreceptor activity by hyperoxia potentially removes the remaining source of afferent chemoreceptor information in the syndrome. Neural sites responsive to hyperoxia should reveal areas mediating central processing to chemoreception free of input from peripheral signaling. The findings will complement data from hypoxia and hypercapnia challenges $(13,14)$.

The objective of this study was to examine neural responses mediating the normal slowing of heart rate and changes in breathing that accompany exposure to high levels of $\mathrm{O}_{2}$, and alterations in these patterns that may mediate the aberrant respiratory and cardiac responses in CCHS. Because hyperoxia is associated with both immediate and late-developing physiologic changes, it was important to follow time courses of neural patterns. We used fMRI procedures to measure changes in BOLD signals to hyperoxic $\left(100 \% \mathrm{O}_{2}\right)$ challenges, because the BOLD signals indirectly relate to neural activity (15). We hypothesized that structures mediating respiratory timing early and late in the challenge would show deficient responses temporally matching the deficits in breathing and heart rate during hyperoxia in CCHS, and that brain areas regulating sympathetic outflow would be similarly affected in the syndrome.

\section{METHODS}

Fourteen children with a diagnosis of CCHS ( 7 male, 7 female) and 15 controls ( 8 male, 7 female) participated. Fourteen pairs were age- and gendermatched; the remaining control subject was matched with one CCHS case (age in years for each group: mean, 11; range, $8-15$; SD 2). Diagnosis was based on standard criteria (16). Patients were ventilated via tracheostomy only during sleep, and not during waking, and showed a clear reduction in ventilatory responses to hypercapnia. Patients with Hirschsprung's disease were excluded, as were patients with additional cardiac, pulmonary, or neuromuscular disorders

Subjects lay supine in an MRI scanner and breathed spontaneously through a two-way nonrebreather valve. Tracheostomy openings were closed throughout the studies. Masking tape across the forehead and foam pads on either side of the head were used to minimize head movement. Measurements of airflow and the ECG were recorded simultaneously with the fMRI signal (3). Each subject underwent two scanning periods, the first consisting of a 150-s baseline and the second a 30-s baseline followed without pause by a 120 -s challenge. A 2-min period was chosen as sufficiently long to elicit early central and peripheral chemoreceptor responses (which occur within the first minute), and to allow certain slower responding changes to occur (second minute). More lengthy challenge periods would have been desirable, but were precluded because of technical limitations with the scanner (newer MRI scanning technology resolves such issues for future studies). The hyperoxia challenge (100\% $\mathrm{O}_{2}$ ) was delivered via the inspiratory arm of the valve throughout the 120-s challenge period.

Images were collected using a 1.5 Tesla scanner (General Electric Signa, GE Medical Systems, Milwaukee, WI). For each 150-s scanning period, 24 volumes of 20 oblique image slices were collected using a gradient echo EPI protocol $\left(\mathrm{TR}=6 \mathrm{~s}, \mathrm{TE}=60 \mathrm{~ms}\right.$, flip angle $=90^{\circ}, \mathrm{FOV}=30 \times 30 \mathrm{~cm}$, no interslice gap, and voxel size $2.3 \times 2.3 \times 5 \mathrm{~mm}$ ). The EPI protocol used the BOLD intrinsic contrast to highlight changes in neural activity during the challenge. Conventional spin echo $\mathrm{T} 1$-weighted images $(\mathrm{TR}=500 \mathrm{~ms}, \mathrm{TE}=$ $9 \mathrm{~ms}, \mathrm{FOV}=30 \times 30 \mathrm{~cm}$, no interslice gap, voxel size $1.2 \times 1.2 \times 5 \mathrm{~mm}$ ) were collected at the same location and orientation to aid in anatomical identification.

The images were preprocessed using the statistical parametric mapping package SPM (17), and custom software. EPI volumes were adjusted for slice timing, motion corrected, and spatially normalized. Segmentation was performed on the spatially normalized images to create a gray matter mask (18). This image mask was applied to the spatially normalized images, which were then smoothed. Global changes were removed (19), and the images were subsequently smoothed and analyzed for significant signal changes.

Two types of analyses were performed on the preprocessed images: 1) VOI analysis using custom routines, and 2) cluster analysis using SPM. VOI analysis allowed the investigation of the response of various structures without assumptions regarding the pattern of response. Additionally, because each VOI was outlined on a subject-by-subject basis, the VOI time trends were not affected by inevitable discrepancies in spatial normalization. The cluster analysis allowed a whole-brain search for regions not selected as VOI, but with the restriction that the response pattern be generally increasing or decreasing during the challenge period.

VOI analysis used a priori defined regions (examples from one subject in Fig. 1), within which the voxel intensities were averaged for each subject. RMANOVA was used to assess both differences from baseline for each group. as well as response differences between the groups (20).

Cluster analysis was performed over the entire brain on a voxel-by-voxel basis, comparing the time course of each voxel to a parametric boxcar model [step function from baseline (off), to challenge (on)], convolved with a standard hemodynamic response function. The fit of such a model was calculated for each subject, with resulting estimates of the contribution of the boxcar pattern at each voxel recorded. Clusters of voxels where group differences between CCHS and control subjects approximately matched this boxcar pattern were identified by performing a two-sample $t$ test of the boxcar estimates at each voxel (from the fMRI literature, a population or random effects analysis). Cluster analysis was used to provide an overview of areas in which response patterns differed between groups. Statistical criteria of $p<$ 0.05 , corrected for multiple comparisons (false discovery rate), were used for an overview of response sites. For selected clusters, the time courses of all voxels within that cluster were extracted and plotted for the two groups.

\section{RESULTS}

Physiology. Physiologic characteristics to the breathing challenge have been described earlier (3). Briefly, respiratory rates initially fell in CCHS patients, but rose in control subjects over the first $20 \mathrm{~s}$ of the challenge, after which they returned to baseline levels until $60 \mathrm{~s}$, when the CCHS patients showed a transient increase versus a transient decrease in the control subjects. Heart rate changes were more muted in CCHS patients both early (first minute increase: $0-40 \mathrm{~s}$ in CCHS, $10-50 \mathrm{~s}$ in controls) and later (second minute decline: from $60 \mathrm{~s}$ in CCHS, $70 \mathrm{~s}$ in controls) to the challenge. Respiratoryrelated heart rate variation was especially reduced in CCHS, and remained well below control levels throughout the challenge.

Global BOLD signal. The global BOLD signal increased in both groups during the later portion of the challenge period (45 $\mathrm{s}$ onwards), but initial (first $20 \mathrm{~s}$ ) values declined in the control group, a trend not found in the CCHS patients (21). Detrending removed all global effects (19).

VOI analysis. Table 1 and Figure 2 show the responses in selected VOI. Cerebellar regions, including the vermis and deep nuclei of the dentate and fastigial areas, increased signal throughout the challenge in both groups, but no group differences appeared. The amygdala response was lateralized, with a transient signal decline in the right amygdala in the control group, but an increase in CCHS patients that was coincident with a marked decline in respiratory rate in the patients. The head of the caudate showed a signal decline in control subjects, but a transient increase in CCHS patients. Lentiform nuclei 


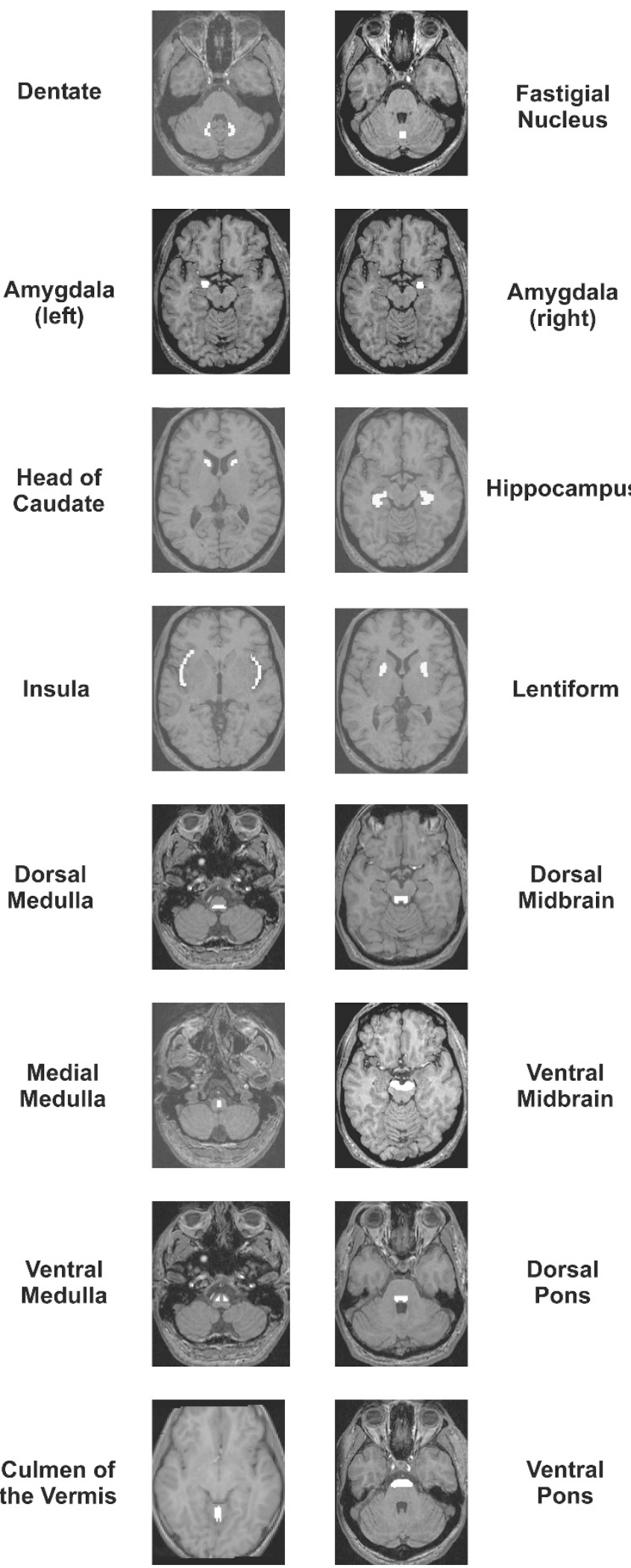

Figure 1. Examples of VOI areas for one subject in white, overlaid onto an individual $\mathrm{T} 1$ image, from which time trends were calculated. A subset of these images was shown earlier for another challenge in a previous publication (34).

showed a transient decline that was more extensive in control than CCHS subjects; both groups returned to baseline after approximately $40 \mathrm{~s}$, with a later decline in both groups, al-
Table 1. Summary of VOI analysis

\begin{tabular}{lccc}
\hline Area name of VOI & $\begin{array}{c}\text { Control } \\
\mathrm{n}=15\end{array}$ & $\begin{array}{c}\text { CCHS } \\
\mathrm{n}=14\end{array}$ & $\begin{array}{c}\text { Group } \\
\text { difference }\end{array}$ \\
\hline Basal ganglia & - & + & $*$ \\
Head of caudate & -- & - & $*$ \\
Lentiform & 0 & 0 & \\
Limbic regions & 0 & - & \\
Amygdala (left) & - & + & $*$ \\
Amygdala (right) & + & + & \\
Hippocampus & + & + & $*$ \\
Insula (left anterior) & + & 0 & $*$ \\
Insula (right anterior) & ++ & + & $*$ \\
Insula (left posterior) & + & 0 & $*$ \\
Insula (right posterior) & & & \\
Brainstem regions & + & -+ & \\
Dorsal midbrain & + & + & $*$ \\
Ventral midbrain & + & + & $*$ \\
Dorsal pons & + & + & \\
Ventral pons & ++ & + & \\
Dorsal medulla & + & 0 & \\
Medial medulla & + & 0 & \\
Ventral medulla & & & \\
Cerebellar regions & + & + & \\
Dentate nucleus & + & + & \\
Fastigial nucleus & + & + & \\
Culmen of the vermis & + & & \\
\hline
\end{tabular}

Significance was tested using RMANOVA $(p<0.05)$. Significant signal increase $(+)$, or decrease $(-)$, or no significant difference over more than one time point is indicated for each group, relative to baseline. Group differences are indicated by an asterisk $(*)$. Where both groups increased and a group effect was noted, the group with the greater extent of signal change is indicated by "++"; similarly, "--" is used for greater extent of decrease, and "-+" for a decline followed by an increase. 0 indicates no significant change from baseline.

though the decline was again more extensive in the control subjects. The hippocampus and insular cortex increased signal throughout the challenge in both groups; however, during the last minute of the challenge in the posterior insula, the control subjects' signal continued to increase to a significantly higher extent than in the CCHS patients. Both groups showed signal increases in the brain stem, with greater enhancement in medullary areas for control subjects, and an initial transient difference in the dorsal midbrain. Midbrain and pontine regions showed initial transient increases in signal.

Cluster analysis. Table 2 shows regions of signal increase throughout the challenge in both groups. Areas within the insula, cerebellar cortex, anterior and medial cingulate, and hippocampus all showed signal increases, together with the dorsomedial nucleus of the thalamus. The region of signal increase was especially extensive in the quadrangular lobule of the cerebellar cortex.

Table 3, and Figures 3 and 4 show regions of group difference in response. Control subjects showed greater signal responses in the medial and anterior cingulate, insula, and caudal hippocampus, along with smaller regions on the right side in the cerebellar cortex, superior temporal gyrus, and inferior frontal gyrus. Regions of greater signal response in CCHS compared with control subjects emerged in the temporal gyrus, pons and midbrain, and left insula and putamen.

Correspondence with physiology. Figure 5 illustrates fMRI signal intensity changes for the right amygdala and posterior 


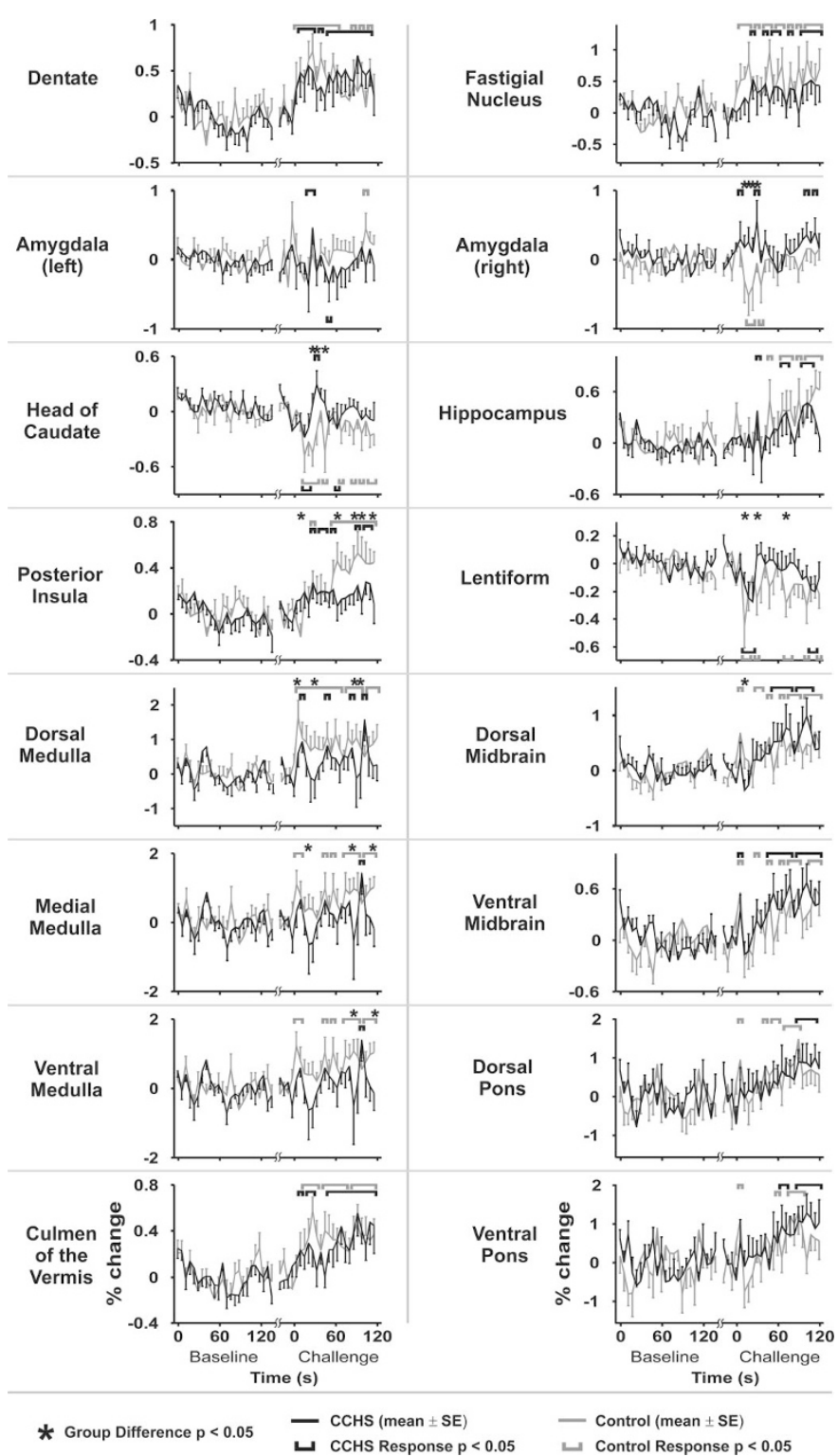

Figure 2. Time trends of VOI from control $(n=15)$ and CCHS $(n=14)$ subjects during baseline and challenge periods, with vertical SE bars. Time points of significant group difference are indicated by $*$ above the plots (RMANOVA, $p<0.05$ ). Time points of significant signal increase or decrease relative to baseline within each group are indicated by horizontal bars above or below plots (key at bottom of figure). Baseline series are plotted on the left of the graphs, and preceded the challenge series by several minutes.

insula overlaid with breathing or heart rate traces for CCHS and control groups. These traces highlight the concurrent alterations in breathing rate with amygdala BOLD signal changes, especially during the first $30 \mathrm{~s}$ of the challenge. The heart rate patterns follow the posterior insula signals, in particular, during the second minute of the challenge.

\section{DISCUSSION}

Hyperoxia elicited distinct response patterns in limbic structures involved in respiratory timing and autonomic outflow, basal ganglia recruited in motor coordination, and pontine and medullary areas targeted by PHOX2B gene expression, mutations of which are suspected of causing autonomic deficits in
Table 2. Clusters of voxels with a significant signal increase throughout the challenge in control and CCHS groups $(p<0.05$, corrected for multiple comparisons)

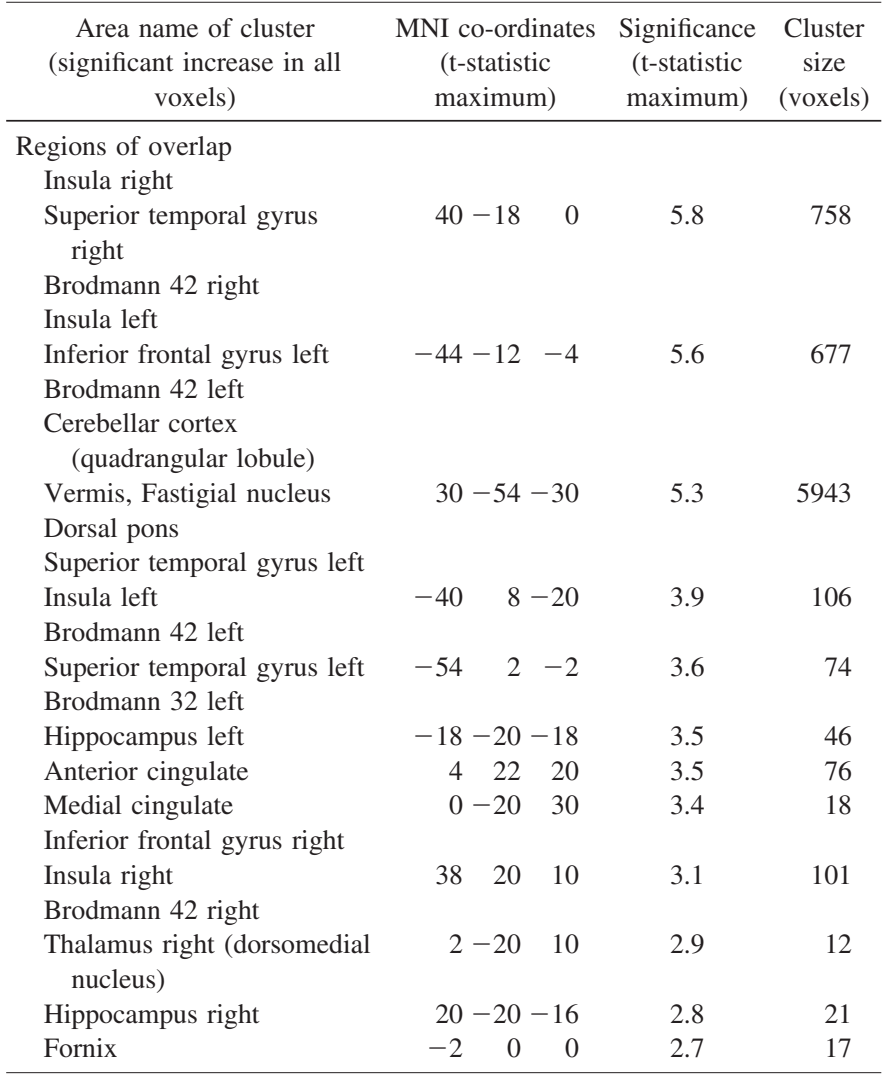

All voxels in these regions exhibited significant signal increases relative to baseline, at the $p<0.05$ level. The t-statistic maximum is an indication of the robustness of the increase at the "best-fit" voxel within the region, or cluster.

CCHS (22-24). Specific response differences temporally coincided with breathing and cardiac rate responses to the challenge.

Interpretation of BOLD signal changes. The BOLD signal is usually interpreted as an indirect indication of the functional processing performed by regions where local signal changes independently of the global signal. An increase in signal reflects increased input and intercellular processing, as opposed to just an increase in neuronal firing rate (25). The BOLD signal is influenced by global cerebral blood flow, blood volume, and oxygenation independently of localized changes resulting from neural activity-related oxygenation demands. The previously described difference in initial global BOLD signal response was indicative of a reduced cerebral autoregulatory capacity in CCHS subjects (21). However, local BOLD signal changes occur in an additive fashion (26), and procedures were implemented to remove all global effects (19). A consequence of the global effects procedure is that any neural signal pattern matching the global pattern would also be removed.

Comparable control and CCHS group responses. The cerebellar responses were similar in both groups and involved an increased signal throughout the challenge, as shown by the VOI and cluster analysis. The similarity of the cerebellar response in both groups is notable, inasmuch as cerebellar 
Table 3. Clusters of voxels with a significant difference in response between groups ( $p<0.05$, corrected for multiple comparisons)

\begin{tabular}{|c|c|c|c|c|}
\hline Area name of cluster & $\begin{array}{l}\text { MNI co-ordinates } \\
\text { (t-statistic maximum) }\end{array}$ & $\begin{array}{c}\text { Significance } \\
\text { (t-statistic maximum) }\end{array}$ & $\begin{array}{l}\text { Cluster size } \\
\text { (voxels) }\end{array}$ & $\begin{array}{c}\text { Control } \\
v s \text { CCHS }\end{array}$ \\
\hline \multicolumn{5}{|l|}{ Regions of overlap } \\
\hline Medial and anterior cingulate & $2 \quad 4 \quad 32$ & 3.2 & 64 & $>$ \\
\hline \multicolumn{5}{|l|}{ Brodmann 42 left } \\
\hline Cerebellar cortex & $12-40-6$ & 3.1 & 21 & $>$ \\
\hline \multicolumn{5}{|l|}{ Parahippocampal gyrus right } \\
\hline \multicolumn{5}{|l|}{ Brodmann 42 right } \\
\hline Insula left & $\begin{array}{lll}-34 & 22 & 0\end{array}$ & 2.8 & 38 & $>$ \\
\hline Parahippocampal gyrus left & $-18-32-4$ & 2.6 & 43 & $>$ \\
\hline Temporal gyrus right & $56-58-2$ & 3.6 & 304 & $<$ \\
\hline Insula left & $-32-12-6$ & 2.7 & 35 & $<$ \\
\hline
\end{tabular}

structures are remarkable in showing different response patterns to a range of ventilatory challenges in other sleepdisordered breathing cases (27), and to cold pressor and expiratory loading challenges in CCHS (28, 28a). Cerebellar sites normally participate in mediating respiratory challenges (29) and are frequently recruited during large changes in blood pressure rather than conditions of minor change (30). The ventilatory and blood pressure challenges in other CCHS studies are associated with substantial elevation of blood pressure. The cardiovascular component in the current study, however, was minimal in extent, and perhaps less likely to reveal cerebellar group differences. The dorsal medial thalamus showed similar response patterns in both groups; a portion of that region projects to ventral respiratory group neurons in the rat (31).

CCHS patients earlier showed significant areas of deficits to hypercapnia and hypoxia in the dorsal medial thalamus and cerebellar cortex and deep nuclei $(13,14)$. The dorsal thalamus shows $c$-fos expression to hypoxia (32), and must be intact for the normal biphasic ventilatory response to hypoxia in the sheep fetus (33). The cerebellar cortex shows deficits to multiple ventilatory and pressor challenges, including hypercapnia (13), hypoxia (14), expiratory loading (28), and cold pressor challenges (28a). The classic role attributed to cerebellar function has been to mediate extremes of afferent input; the structure normally does not regulate momentary blood pressure changes, for example, but is involved in restorative action to extreme hypo- or hypertension (30). The cerebellum may play similar compensatory roles in hypercapnia and hypoxia, both of which are life-threatening. In extreme blood pressure and ventilatory challenges, the cerebellar role may be one of compensatory respiratory motor or other somatomotor action to restore perfusion or appropriate ventilation. Hyperoxia is not immediately life-threatening, and we speculate that cerebellar structures may not serve comparable regulatory roles for high $\mathrm{O}_{2}$ stimulation. The dorsal thalamic role may serve comparable survival functions, but from arousal processes. Both hypercap- nic and hypoxic challenges elicited a near-continuous column of deficient responses from the thalamus to pontine regions near the locus coeruleus $(13,14)$, the latter a site with widespread alerting processes (36). We speculate that dorsal thalamic responses to hypercapnia and hypoxia, which show deficits in CCHS patients to these two challenges but not to hyperoxia, reflect a survival-related chemosensitivity response.

Initial responses-group differences. The amygdala responses in CCHS were unique in their timing and lateralization. These responses were inverse to those of control subjects and paralleled the opposite respiratory rate pattern between groups found early in the challenge. The timing of response pattern differences raises the possibility of a role for amygdala structures in mediating the slowing of respiratory rate in CCHS over the increased rate in control subjects during that initial period. The amygdala is not normally considered a "respiratory-related" structure, but can modify respiratory rate, because the central amygdala nucleus projects heavily to respiratory phase-switching areas of the dorsal pons (37), and, on singlepulse stimulation, can pace the respiratory rhythm, a dependency that declines with entry into sleep (38); projections from the amygdala/hippocampal region have also been demonstrated to the ventral respiratory region in the rat (31). A role for the amygdala in breathing control in CCHS is especially of interest, inasmuch as CCHS patients typically do not readily perceive "air hunger" from increasing $\mathrm{CO}_{2}$ (39) or potential asphyxia, and do not show signs of emotional distress to the condition; affected patients must be encouraged to voluntarily breathe when very relaxed, despite extreme $\mathrm{O}_{2}$ desaturation. The reduced anxiety in CCHS patients extends to other behavioral stimuli, as assessed by neuropsychological examination (40). Amygdala structures have long been known to mediate distress or negative emotion (for overview, see 41), and has been implicated, together with cingulate, cerebellar, and insular structures, with dyspnea $(42,43)$, and the structure has considerable potential to enhance switching to inspiratory effort on activation. Aberrant function in this site to the chal- 
C
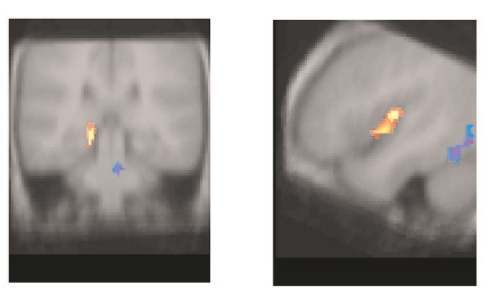

2
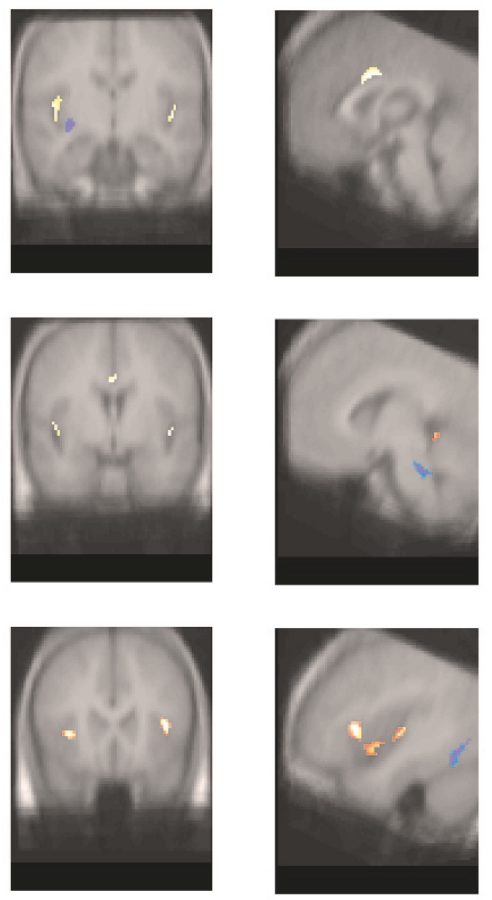

S1...4

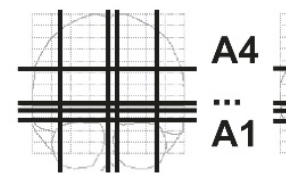

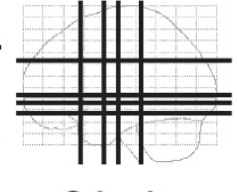

C4...1
A
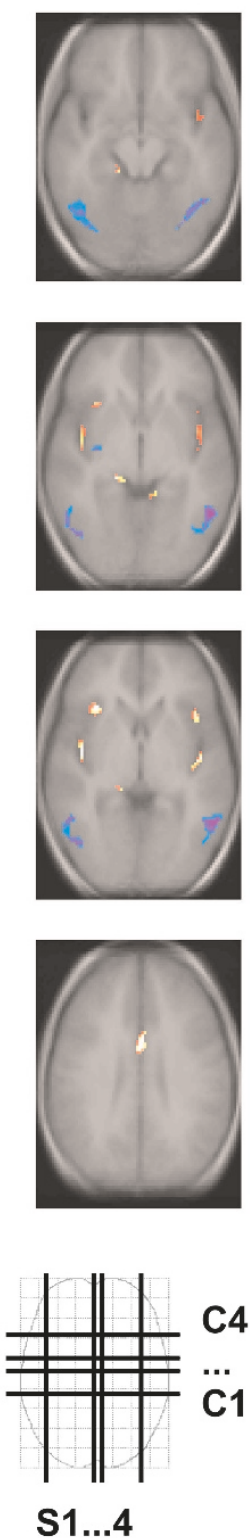

S1...4 (t)

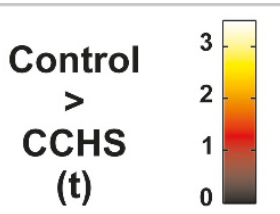

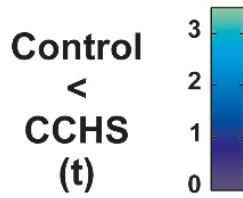

(t)
Remarkably, the role of the amygdala in mediating aversive conditioning appears to be preferentially lateralized to the right side in rodents $(46,47)$, and at least partially on the right side in humans (48). It is unclear whether the differential CCHS response to hyperoxia reflects deficiencies in the normal lateralized function for this structure, or whether influences from other related areas (e.g. right insula, right cingulate) may contribute to the findings.

Several of these sites, especially the dorsal medulla and dorsal pons, are targets for PHOX2B gene expression; a mutation of PHOX2B is found in a substantial proportion of CCHS patients $(22,24)$. The gene is especially involved in autonomic nervous system development, and particularly in a sensory structure involved in afferent detection of $\mathrm{O}_{2}$ levels, the NTS, and an area involved in respiratory phase-switching and blood pressure control, the dorsal pons (23). The dorsal pontine differences consisted of higher responses in CCHS patients. Both white matter damage and diminished cell density have been noted for medullary structures in the syndrome $(49,50)$.

Sustained group differences-clusters. Several areas showed patterns of differing responses between groups that emerged early, and were sustained throughout the entire challenge. These areas included the posterior temporal cortex, insula, medial cingulate, and caudal and dorsal hippocampus (Figs. 3 and 4). The response pattern was typically one of no or little change in the CCHS group, and an increase or decline in the control subjects, although in the hippocampus, a late rise emerged in CCHS patients over an initial transient response in control subjects, and transient patterns in the opposite direction developed in the dorsal pons. The data illustrate the extent of "unreactive" areas in CCHS patients, despite substantial signal increases in other areas.

Longer-latency, first- or second-minute group response differences. The lentiform nuclei showed an initial response that was greater in control than CCHS subjects, followed by a later signal decline in both groups. Again, the response was more extensive in the control than the CCHS patients. Response patterns also differed in the head of the caudate nucleus. Both areas within the basal ganglia are involved in motor coordination, presumably affecting respiratory muscle patterning.

Remarkable among rostral brain areas, the anterior and posterior insula showed much-reduced responses in CCHS late in the challenge over control findings. The data reinforce a central role for insular structures to mediate autonomic outflow, a relationship earlier outlined by others (51-53), with lateralization of sympathetic modulation by the right insula, and parasympathetic action by the left side. Portions of the insula exert modulatory control over baroreflexes (54). The absence of a response in CCHS relative to control subjects was reflected as a more pronounced difference on the right (sympathetic) side, and was particularly enhanced late in the challenge (Fig. 4). Heart rate slowing was prominent late in the response in control subjects, and minimized in CCHS patients (3). Because the right insula can exert an inhibitory influence on sympathetic outflow (55), unresponsiveness in that site would lead to the physiologic response found here. sponses to both ventilatory and autonomic challenges $(44,45)$. lenge, manifested as an increase in activity rather than the normal transient decline, suggests a failure to appropriately process information on the hyperoxic stimulus.

The amygdala signal response differences emerged only on the right side. A lateralization in multiple brain structures, including the amygdala, has been noted in normal brain re-
Figure 3. Regions of significant group difference $(p<0.05)$ in response control over CCHS subjects color coded in yellow-red scale, and regions of decreased signal in control compared with CCHS subjects color coded in blue-green (key at bottom of figure). 
Control > CCHS
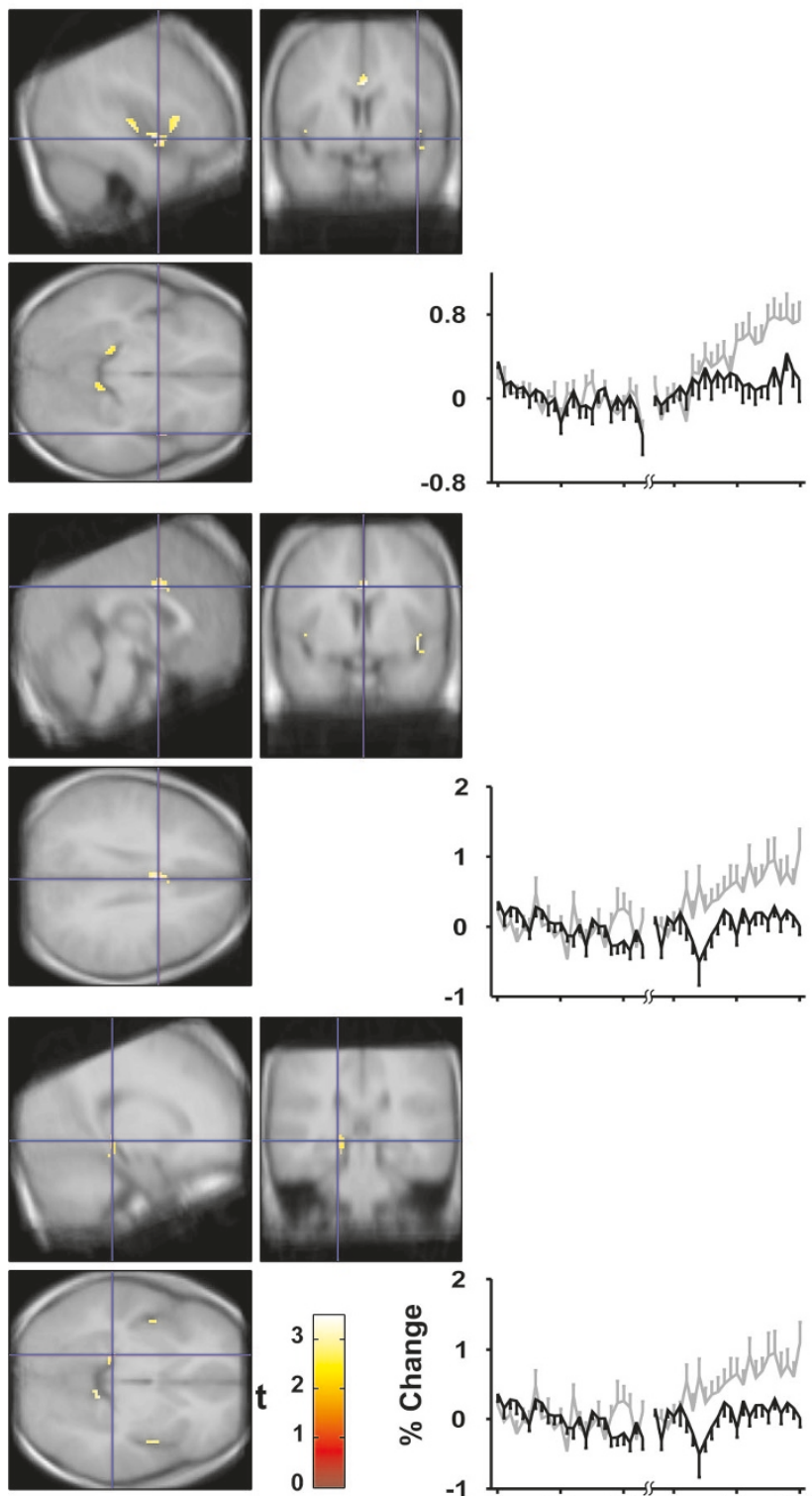

CCHS > Control
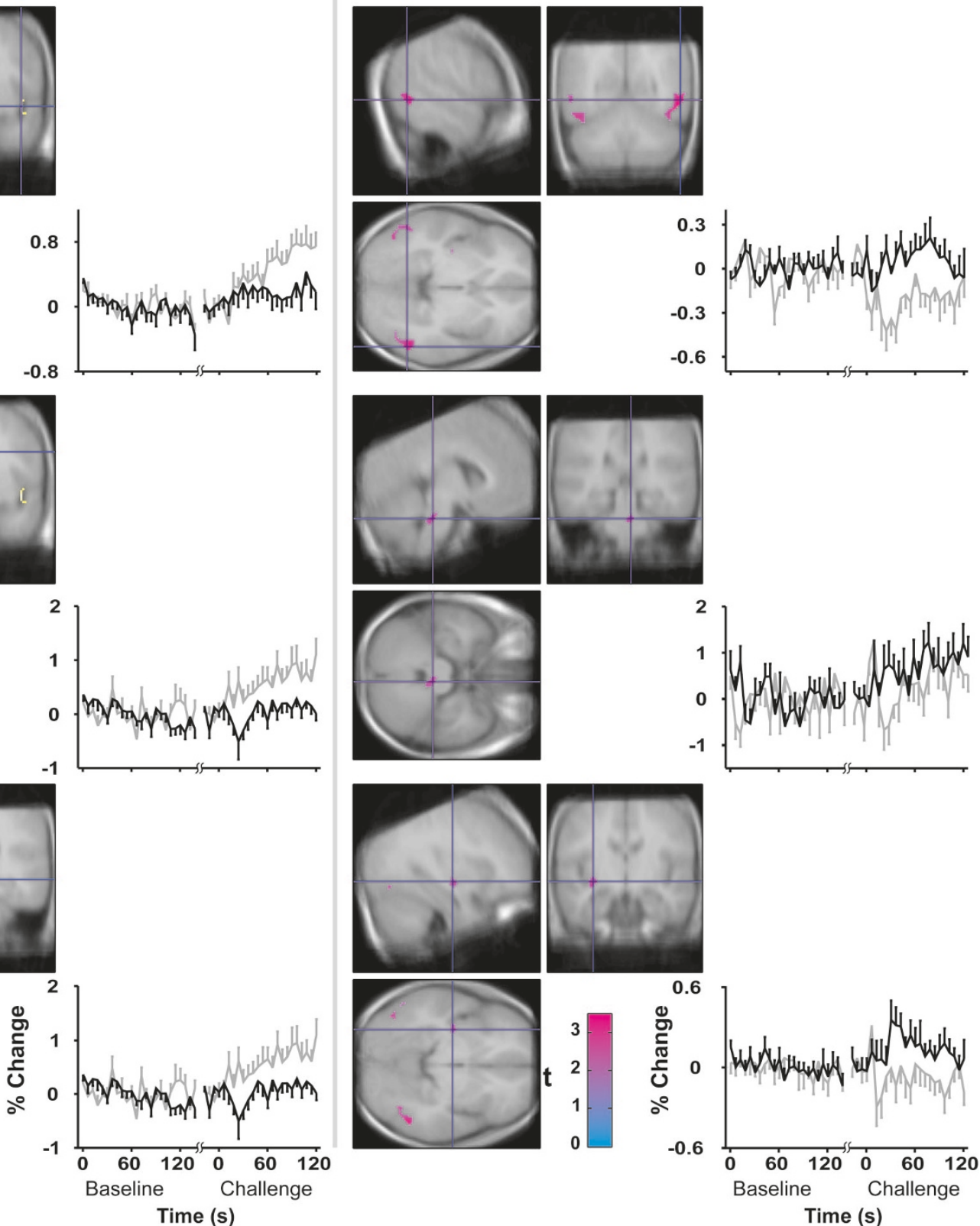

Time (s)

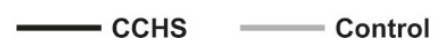

Figure 4. Time trends for control $(n=15)$ and CCHS $(n=14)$ groups from selected clusters of significant group difference $(p<0.05)$, with SE bars; overlays follow the same conventions as Figure 2.

As with respiratory rate responses, the fMRI signal patterns differed between the first and second minutes of the challenge in several regions. After initial transient increases, signals in the dorsal and ventral pons increased only late in the challenge, and presumably reflect the slowing of heart and respiratory rate in both groups. No group difference in pontine signals emerged, despite the significant late physiologic differences in CCHS cases.

Other considerations. The CCHS patients were all ventilator dependent during sleep but not during waking, and thus were a subset of the entire CCHS population, some of whom require continuous ventilation and presumably have more se- rious neural injury. The findings here might be more extensive or differ in other fashions in more seriously affected patients, and that possibility should be explored. The potential for more extensive damage to elicit additional deficits cautions against extension of these findings to the broader CCHS population.

Baseline neural conditions cannot be measured using the technique used, because fMRI procedures provide an index of change rather than absolute measures of functional integrity in particular areas. Neural structures mediating cardiac and respiratory integration are clearly deficient in waking, baseline conditions, as shown by earlier evaluation of respiratory effects on heart rate variation $(3,56)$. The presence of damage in white 


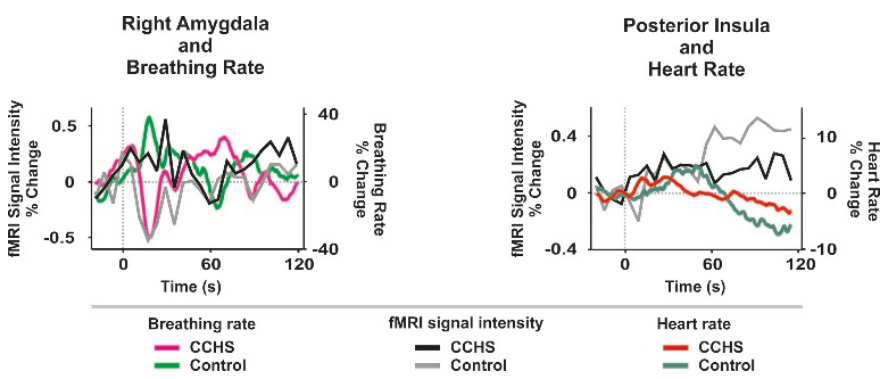

Figure 5. fMRI signal intensity time trends overlaid with breathing (right amygdala) and heart rate (posterior insula) traces for control and CCHS groups; key at bottom. The rate curves are a moving mean (10-s window). The vertical gray dashed line indicates the onset of the challenge. Each graph has a signal intensity scale (left) and a rate scale (right). The breathing and heart rate trend lines are from a previous publication (3).

matter and diminished cell density $(49,50)$ may contribute both to the waking deficits and sleep-related pathology. The current findings add support to evidence that breathing control mechanisms in CCHS are impaired in the waking state as well as during sleep.

Several of the areas affected differentially in CCHS cases by hyperoxia, including sites within the insula and caudate nucleus, are not structures described as targeted by PHOX2B gene expression. In addition to hyperoxia, these areas consistently show deficits to other ventilatory and pressor challenges $(13,14,27,28 \mathrm{a})$. It is unclear why these areas are affected in the syndrome; some of the structures, such as the basal ganglia, are susceptible to intermittent hypoxia, but others are not. It may be the case that PHOX2B expression extends to structures in addition to autonomic ganglia, medullary, and pontine sites originally described (23). Undetected mutations, other than PHOX2B, may also be operating in CCHS, but this possibility remains speculative.

Processes. The nature of the processes by which structures show deficits to hyperoxia could follow several mechanisms. The defects could be primarily peripheral, at the receptor or afferent pathway level. However, CCHS patients show at least partially intact peripheral chemoreceptor responses (6), thus the response deficits likely result from failure of central reaction to high levels of $\mathrm{O}_{2}$. Hyperoxia should suppress peripheral chemoreceptor activity. The absence of chemoreceptor traffic constitutes as much of a signal to central neural structures as differing levels of input, and presumably results in the recruitment of neural responses found in control subjects and common areas in multiple sites of CCHS patients. The failure of select central sites, or the failure to send appropriate information to multiple more-rostral areas, particularly those mediating cardiovascular regulation, such as the insula, frontal cortex, and insula, appear to underlie the most likely defective processes. The response deficits to hyperoxia, therefore, are complex, and appear to reflect selective interactive failure of signaling between structures.

\section{SUMMARY}

Hyperoxia elicited comparable responses in CCHS and control subjects in multiple areas of the brain, and distinctly different patterns in other regions. The response differences emerged both early and late in the challenge, and in sites that affect timing of respiratory switching, such as the amygdala, or respiratory and cardiovascular concomitants of the challenge, such as the insula, cingulate, and dorsal and ventral medulla. In several of these areas, including the insula, the response pattern was largely "no change" in CCHS cases, whereas signals increased or declined in control subjects. The findings are remarkable in demonstrating apparent significant roles for limbic sites in mediating respiratory and cardiovascular patterns to hyperoxia, and an apparent absence of cerebellar response differences in CCHS patients, unlike other challenges that elicit larger blood pressure effects.

Acknowledgments. The authors thank Dr. Munawar Saeed, Ms. Amy Kim, and Ms. Claire Valderama for technical support, and Dr. Rajesh Kumar for editorial assistance.

\section{REFERENCES}

1. Haddad GG, Mazza NM, Defendini R, Blanc WA, Driscoll JM, Epstein MA, Epstein RA, Mellins RB 1978 Congenital failure of automatic control of ventilation, gastrointestinal motility and heart rate. Medicine (Baltimore) 57:517-526

2. Commare MC, Francois B, Estournet B, Barois A 1993 Ondine's curse: a discussion of five cases. Neuropediatrics 24:313-318

3. Macey PM, Valderama C, Kim AH, Woo MA, Gozal D, Keens TG, Harper RK, Harper RM 2004 Temporal trends of cardiac and respiratory responses to ventilatory challenges in congenital central hypoventilation syndrome. Pediatr Res 55:953-959

4. Oren J, Kelly DH, Shannon DC 1987 Long-term follow-up of children with congenital central hypoventilation syndrome. Pediatrics 80:375-380

5. Paton JY, Swaminathan S, Sargent CW, Keens TG 1989 Hypoxic and hypercapnic ventilatory responses in awake children with congenital central hypoventilation syndrome. Am Rev Respir Dis 140:368-372

6. Gozal D, Marcus CL, Shoseyov D, Keens TG 1993 Peripheral chemoreceptor function in children with the congenital central hypoventilation syndrome. J Appl Physiol 74:379-387

7. Woo MS, Woo MA, Gozal D, Jansen MT, Keens TG, Harper RM 1992 Heart rate variability in congenital central hypoventilation syndrome. Pediatr Res 31:291-296

8. Trang H, Boureghda S, Denjoy I, Alia M, Kabaker M 2003 24-hour BP in children with congenital central hypoventilation syndrome. Chest 124:1393-1399

9. O'Sullivan J, Cottrell AJ, Wren C 1993 Ondine's curse and neurally mediated syncope - a new and important association. Eur Heart J 14:1289-1291

10. Silvestri JM, Hanna BD, Volgman AS, Jones PJ, Barnes SD, Weese-Mayer DE 2000 Cardiac rhythm disturbances among children with idiopathic congenital central hypoventilation syndrome. Pediatr Pulmonol 29:351-358

11. Weese-Mayer DE, Silvestri JM, Menzies LJ, Morrow-Kenny AS, Hunt CE, Hauptman SA 1992 Congenital central hypoventilation syndrome: diagnosis, management, and long-term outcome in thirty-two children. J Pediatr 120:381-387

12. Dejours P 1962 Chemoreflexes in breathing. Planta Med 42:335-358

13. Harper RM, Macey PM, Woo MA, Macey KE, Keens TG, Gozal D, Alger J 2005 Hypercapnic exposure in Congenital Central Hypoventilation Syndrome reveals central nervous system respiratory control mechanisms. J Neurophysiol 93:16471658

14. Macey PM, Woo MA, Macey KE, Keens TG, Saeed MM, Alger JR, Harper RM 2004 Hypoxia reveals posterior thalamic, cerebellar, midbrain and limbic deficits in Congenital Central Hypoventilation Syndrome. J Appl Physiol 98:958-969

15. Ogawa S, Lee TM, Kay AR, Tank DW 1990 Brain magnetic resonance imaging with contrast dependent on blood oxygenation. Proc Natl Acad Sci U S A 87:9868-9872

16. American Thoracic Society 1999 Idiopathic congenital central hypoventilation syndrome: diagnosis and management. Am J Respir Crit Care Med 160:368-373

17. Friston KJ, Holmes AP, Poline JB, Grasby PJ, Williams SC, Frackowiak RS, Turner R 1995 Analysis of fMRI time-series revisited. Neuroimage 2:45-53

18. Ashburner J, Friston K 1997 Multimodal image coregistration and partitioning-a unified framework. Neuroimage 6:209-217

19. Macey PM, Macey KE, Kumar R, Harper RM 2004 A method for removal of global effects from fMRI time series. Neuroimage 22:360-366

20. Littell RC, Milliken GA, Stroup WW, Wolfinger RD 1996 SAS System for Mixed Models. SAS Institute Inc., Cary, NC

21. Macey PM, Alger JR, Kumar R, Macey KE, Woo MA, Harper RM 2003 Global BOLD MRI changes to ventilatory challenges in congenital central hypoventilation syndrome. Respir Physiol Neurobiol 139:41-50

22. Amiel J, Laudier B, Attie-Bitach T, Trang H, de Pontual L, Gener B, Trochet D, Etchevers H, Ray P, Simonneau M, Vekemans M, Munnich A, Gaultier C, Lyonnet S 2003 Polyalanine expansion and frameshift mutations of the paired-like homeobox gene PHOX2B in congenital central hypoventilation syndrome. Nat Genet 33:459461

23. Dauger S, Pattyn A, Lofaso F, Gaultier C, Goridis C, Gallego J, Brunet JF 2003 Phox $2 \mathrm{~b}$ controls the development of peripheral chemoreceptors and afferent visceral pathways. Development 130:6635-6642 
24. Weese-Mayer DE, Berry-Kravis EM, Zhou L, Maher BS, Silvestri JM, Curran ME, Marazita ML 2003 Idiopathic congenital central hypoventilation syndrome: analysis of genes pertinent to early autonomic nervous system embryologic development and identification of mutations in PHOX2b. Am J Med Genet 123A:267-278

25. Logothetis NK, Pauls J, Augath M, Trinath T, Oeltermann A 2001 Neurophysiological investigation of the basis of the fMRI signal. Nature 412:150-157

26. Corfield DR, Murphy K, Josephs O, Adams L, Turner R 2001 Does hypercapniainduced cerebral vasodilation modulate the hemodynamic response to neural activation? Neuroimage 13:1207-1211

27. Macey PM, Macey KE, Henderson LA, Alger JR, Frysinger RC, Woo MA, Yan-Go F, Harper RM 2003 Functional magnetic resonance imaging responses to expiratory loading in obstructive sleep apnea. Respir Physiol Neurobiol 138:275-290

28. Macey KE, Macey PM, Woo MA, Harper RK, Alger JR, Keen TG, Harper RM 2004 fMRI signal changes to forced expiratory loading in congenital central hypoventilation syndrome. J Appl Physiol 97:1897-1907

28a. Macey PM, Macey KE, Woo MA, Keens TG, Harper RM 2005 Aberrant neura responses to cold pressor challenges in congenital central hypoventilation syndrome. Pediatr Res 57:500-509

29. Xu F, Frazier DT 2002 Role of the cerebellar deep nuclei in respiratory modulation. Cerebellum 1:35-40

30. Lutherer LO, Lutherer BC, Dormer KJ, Janssen HF, Barnes CD 1983 Bilatera lesions of the fastigial nucleus prevent the recovery of blood pressure following hypotension induced by hemorrhage or administration of endotoxin. Brain Res 269:251-257

31. Gaytan SP, Pasaro R 1998 Connections of the rostral ventral respiratory neuronal cell group: an anterograde and retrograde tracing study in the rat. Brain Res Bull 47:625-642

32. Sica AL, Greenberg HE, Scharf SM, Ruggiero DA 2000 Chronic-intermitten hypoxia induces immediate early gene expression in the midline thalamus and epithalamus. Brain Res 883:224-228

33. Koos BJ, Chau A, Matsuura M, Punla O, Kruger L 1998 Thalamic locus mediates hypoxic inhibition of breathing in fetal sheep. J Neurophysiol 79:2383-2393

34. Deleted in proof

35. Deleted in proof

36. Aston-Jones G, Rajkowski J, Kubiak P, Valentino RJ, Shipley MT 1996 Role of the locus coeruleus in emotional activation. Prog Brain Res 107:379-402

37. Hopkins DA, Holstege G 1978 Amygdaloid projections to the mesencephalon, pons and medulla oblongata in the cat. Exp Brain Res 32:529-547

38. Harper RM, Frysinger RC, Trelease RB, Marks JD 1984 State-dependent alteration of respiratory cycle timing by stimulation of the central nucleus of the amygdala. Brain Res 306:1-8
39. Shea SA, Andres LP, Shannon DC, Guz A, Banzett RB 1993 Respiratory sensations in subjects who lack a ventilatory response to $\mathrm{CO}_{2}$. Respir Physiol 93:203-219

40. Pine DS, Weese-Mayer DE, Silvestri JM, Davies M, Whitaker AH, Klein DF 1994 Anxiety and congenital central hypoventilation syndrome. Am J Psychiatry $151: 864-870$

41. Amaral DG 2003 The amygdala, social behavior, and danger detection. Ann N Y Acad Sci 1000:337-347

42. Evans KC, Banzett RB, Adams L, McKay L, Frackowiak RS, Corfield DR 2002 BOLD fMRI identifies limbic, paralimbic, and cerebellar activation during air hunger. J Neurophysiol 88:1500-1511

43. Peiffer C, Poline JB, Thivard L, Aubier M, Samson Y 2001 Neural substrates for the perception of acutely induced dyspnea. Am J Respir Crit Care Med 163:951-957

44. Harper RM, Bandler R, Spriggs D, Alger JR 2000 Lateralized and widespread brain activation during transient blood pressure elevation revealed by magnetic resonance imaging. J Comp Neurol 417:195-204

45. King AB, Menon RS, Hachinski V, Cechetto DF 1999 Human forebrain activation by visceral stimuli. J Comp Neurol 413:572-582

46. Baker KB, Kim JJ 2004 Amygdalar lateralization in fear conditioning: evidence for greater involvement of the right amygdala. Behav Neurosci 118:15-23

47. Scicli AP, Petrovich GD, Swanson LW, Thompson RF 2004 Contextual fear conditioning is associated with lateralized expression of the immediate early gen -fos in the central and basolateral amygdalar nuclei. Behav Neurosci 118:5-14

48. Morris JS, Dolan RJ 2004 Dissociable amygdala and orbitofrontal responses during reversal fear conditioning. Neuroimage 22:372-380

49. Liu HM, Loew JM, Hunt CE 1978 Congenital central hypoventilation syndrome: a pathologic study of the neuromuscular system. Neurology 28:1013-1019

50. Ozawa Y, Okado N 2002 Alteration of serotonergic receptors in the brain stems of human patients with respiratory disorders. Neuropediatrics 33:142-149

51. Allen GV, Saper CB, Hurley KM, Cechetto DF 1991 Organization of visceral and limbic connections in the insular cortex of the rat. J Comp Neurol 311:1-16

52. Oppenheimer SM, Cechetto DF 1990 Cardiac chronotropic organization of the rat insular cortex. Brain Res 533:66-72

53. Oppenheimer SM, Gelb A, Girvin JP, Hachinski VC 1992 Cardiovascular effects of human insular cortex stimulation. Neurology 42:1727-1732

54. Zhang ZH, Rashba S, Oppenheimer SM 1998 Insular cortex lesions alter baroreceptor sensitivity in the urethane-anesthetized rat. Brain Res 813:73-81

55. Butcher KS, Cechetto DF 1995 Autonomic responses of the insular cortex in hypertensive and normotensive rats. Am J Physiol 268:R214-R222

56. Kim AH, Macey PM, Woo MA, Yu PL, Keens TG, Gozal D, Harper RM 2002 Cardiac responses to pressor challenges in congenital central hypoventilation syndrome. Somnologie 6:109-115 